Published in final edited form as:

Nat Neurosci. 2007 June ; 10(6): 787-791.

\title{
Anticipation of monetary gain but not loss in healthy older adults
}

\author{
Gregory R Samanez-Larkin ${ }^{1}$, Sasha E B Gibbs ${ }^{1,2}$, Kabir Khanna ${ }^{1}$, Lisbeth Nielsen ${ }^{3}$, Laura L \\ Carstensen $^{1,4}$, and Brian Knutson ${ }^{1,5}$ \\ 1 Department of Psychology, Stanford University, Jordan Hall, Building 420, Stanford, California \\ 94305-2130, USA
}

2 Helen Wills Neuroscience Institute, University of California Berkeley, 132 Barker Hall, Berkeley, California 94720-3190, USA

3 National Institute on Aging and National Institutes of Health, 7201 Wisconsin Avenue, \#533, Bethesda, Maryland 20892-9205, USA

4 Stanford Center on Longevity, Encina Hall, 616 Serra Street, Stanford, California 94305-6055, USA

5 Neuroscience Institute, Stanford University, Stanford, California 94305, USA

\section{Abstract}

\begin{abstract}
Although global declines in structure have been documented in the aging human brain, little is known about the functional integrity of the striatum and prefrontal cortex in older adults during incentive processing. We used event-related functional magnetic resonance imaging to determine whether younger and older adults differed in both self-reported and neural responsiveness to anticipated monetary gains and losses. The present study provides evidence for intact striatal and insular activation during gain anticipation with age, but shows a relative reduction in activation during loss anticipation. These findings suggest that there is an asymmetry in the processing of gains and losses in older adults that may have implications for decision-making.
\end{abstract}

Over the past several decades, scientists have made rapid progress toward determining the effects of aging on cognition. Both behavioral and neuroimaging studies show that there is a strong negative relationship between age and cognitive performance across many types of tasks ${ }^{1,2}$. However, a growing body of research also suggests that many affective abilities do not decline with age, and that in some cases they may improve. Accumulating behavioral evidence suggests that older adults perform relatively better on tasks that involve the processing of emotional stimuli ${ }^{3}$. Socioemotional selectivity theory postulates that age-related attempts to optimize emotional well-being ${ }^{4}$ generate increased positive emotional experiences and/or decreased negative emotional experiences over the life $\operatorname{span}^{5}$. To date, however, very few neuroimaging studies have focused on changes in emotion with age 6,7 , with only one prior study examining changes in incentive processing over the life span ${ }^{8}$.

Studies of brain structure and chemistry provide some evidence for age-related decline. These studies have specifically shown significant structural atrophy of the caudate, insula and prefrontal cortex, as well as global declines in dopamine receptors in the striatum and the

Correspondence should be addressed to G.S.L. (glarkin@stanford.edu). AUTHOR CONTRIBUTIONS

G.S.L., S.G., L.N., L.C. and B.K. designed the experiment. G.S.L., S.G. and K.K. collected and analyzed the data. All of the authors contributed to the preparation of the manuscript.

COMPETING INTERESTS STATEMENT

The authors declare no competing financial interests.

Reprints and permissions information is available online at http://npg.nature.com/reprintsandpermissions 
prefrontal cortex ${ }^{9-12}$. Currently, the implications of these anatomical and chemical changes for brain function during incentive processing remain unclear.

The monetary incentive delay (MID) task ${ }^{13}$ is designed to elicit both affective responses and neural activation in mesolimbic regions during incentive processing. Event-related functional magnetic resonance imaging (fMRI) studies that have used this task in adolescents and younger adults have implicated striatal and insular activation in the anticipation of uncertain gains and losses ${ }^{14,15}$. Because healthy older adults report preserved (or even enhanced) positive affective experience relative to younger adults on a day-to-day basis ${ }^{16}$, we predicted that both subjective responses and neural activation in anticipation of rewards would be preserved in a healthy older sample. We compared subjective and neural responses to incentives between healthy younger and older adults.

\section{RESULTS}

\section{Self-reported affect}

Younger and older adults reported similar subjective responses during anticipation of gains, but differed during anticipation of losses. An analysis of variance (ANOVA) conducted on cue-elicited affect yielded a significant three-way valence (gain, loss) by magnitude ( $\$ 0.00$, $\$ 0.50, \$ 5.00)$ by age (young, old) interaction $\left(F_{2,21}=9.142, P=0.001\right)$, indicating that the younger and older adults differed in their ratings of gain and loss cues (Fig. 1, Supplementary Fig. 1 online). Within-group ANOVAs (corrected for four comparisons, $P<0.013$ ) revealed significant main effects of magnitude on positive arousal ratings for gain cues $\left(F_{2,10}=34.59\right.$, $P<0.0005)$ and negative arousal ratings for loss cues $\left(\mathrm{F}_{2,10}=39.492, P<0.0005\right)$ in younger adults. Older adults showed a comparable magnitude effect on positive arousal ratings for gain cues $\left(F_{2,10}=29.564, P<0.0005\right)$, but a weaker, albeit still significant, magnitude effect on negative arousal for loss cues $\left(F_{2,10}=9.825, P<0.013\right)$. Between-group comparisons indicated that younger adults reported greater negative arousal for large loss cues $(\$ 5.00)$ than did older adults $\left(T_{22}=5.90, P<0.008\right)$, but ratings for the other cues did not significantly differ (all $P$ $>0.008$ ). See Supplementary Results online for further analyses of self-reported affect.

\section{Neural activity}

Localization analyses confirmed that during gain anticipation, both younger and older adults showed significant ventral striatal, medial caudatal and anterior insular activation at the global threshold $(P<0.0001$; Supplementary Fig. 2 and Supplementary Table 1 online). During loss anticipation, younger adults showed significant medial caudatal and anterior insular activation at the global threshold $(P<0.0001$; Supplementary Fig. 3 online), but showed ventral striatal activation only at the small-volume-corrected threshold $(P<0.005)$. Older adults showed anterior insular activation only at the small-volume-corrected threshold $(P<0.005)$. Betweengroup $t$-tests revealed no differences during gain anticipation, and also showed that younger adults had greater activation of medial caudate and anterior insula during loss anticipation at the small-volume-corrected threshold ( $P<0.005 ;$ Fig. 2 and Supplementary Table 2 online).

Volume of interest (VOI) analyses confirmed that although both younger and older adults activated the ventral striatum, medial caudate (MCAUD) and anterior insula (AINS) during gain anticipation, only younger adults showed significant MCAUD and AINS activation during loss anticipation.

A mixed-model ANOVA of anticipatory activation in the right ventral striatum yielded a significant interaction of valence and magnitude $\left(F_{2,21}=3.916, P<0.05\right)$, but a nonsignificant interaction of valence, magnitude and age $\left(F_{2,21}=1.50, P=0.25\right)$, suggesting that activation in the ventral striatum was greater for gain than for loss anticipation, and did not differ between 
younger and older participants (Supplementary Results, Supplementary Discussion and Supplementary Figs. 4 and 5 online). Cue-elicited affect was also correlated with activation in the ventral striatum across individuals. Self-reported positive arousal correlated with activation during gain anticipation $(R=0.42, P<0.05$; Supplementary Figs. 6 and 7 online), but selfreported negative arousal did not correlate with activation during loss anticipation $(R=0.21$, $P=0.16$; Supplementary Results).

A mixed-model ANOVA of anticipatory activation in the left MCAUD yielded a significant three-way interaction of valence, magnitude and age $\left(F_{2,21}=5.35, P<0.05\right.$; Fig. 3$)$. Withingroup ANOVAs (corrected for four comparisons, $P<0.013$ ) revealed significant linear main effects of magnitude on MCAUD activation for gain $\left(F_{2,10}=8.44, P<0.001\right)$ and loss cues $\left(F_{2,10}=20.40, P<0.0005\right)$ in younger adults. Older adults, however, showed a significant linear magnitude effect for gain cues $\left(F_{2,10}=15.82, P<0.005\right)$, but not loss cues $\left(F_{2,10}=1.07\right.$, $P=0.38)$. For full activation time courses see Supplementary Figure 8 online. Cue-elicited affect was also correlated with activation in the left MCAUD across individuals. Self-reported positive arousal did not correlate with caudatal activation during gain anticipation $(R=-0.01$, $P=0.47)$, but self-reported negative arousal correlated with caudatal activation during loss anticipation $(R=0.42, P<0.05$; Supplementary Fig. 6).

A mixed-model ANOVA of anticipatory activation in the right AINS yielded a significant three-way interaction of valence, magnitude and age $\left(F_{2,21}=3.95, P<0.05\right)$ (Fig. 4). Withingroup ANOVAs (corrected for four comparisons, $P<0.013$ ) revealed significant linear main effects of magnitude on AINS activation for gain $\left(F_{2,10}=14.549, P<0.005\right)$ and loss cues $\left(F_{2,10}=20.571, P<0.005\right)$ in younger adults. Older adults, however, showed a significant linear magnitude effect for gain cues $\left(F_{2,10}=71.351, P<0.0005\right)$ but not loss cues $\left(F_{2,10}=\right.$ $1.546, P=0.24)$. For full activation time courses see Supplementary Figure 9 online. Cueelicited affect was also correlated with activation in the right AINS across individuals. Selfreported positive arousal correlated with insular activation during gain anticipation $(R=0.41$, $P<0.05$; Supplementary Fig. 6), and self-reported negative arousal correlated with insular activation during loss anticipation $(R=0.38, P<0.05$; Supplementary Fig. 6$)$.

For analyses of neural activity during incentive outcomes see Supplementary Results and Supplementary Figures 10, 11, and 12 online.

\section{DISCUSSION}

Neither self-reported affect nor brain activation data yielded evidence of a difference between younger and older adults during gain anticipation, but both suggested a difference between these groups during loss anticipation. Our neuroimaging findings add at least two significant contributions to our self-report findings. First, little is presently known about mesolimbic function in older adults during basic incentive processing tasks, and functional neuroimaging affords a first glimpse at how activation in these regions may be affected by age. Second, many behavioral studies suggest that healthy older adults report reduced experience of negative emotions. The present findings provide physiological evidence suggesting that these age differences may not purely reflect biases in self-reports, although future research will have to further clarify the relationship between neural activation and self-reported emotion.

The lack of differences between younger and older adults in ventral striatal activation during gain anticipation may seem surprising in light of documented age-related impairments on reward reversal learning tasks. For instance, relative to younger adults, a previous study found reduced ventral striatal activation in older adults engaged in a reward reversal learning $\operatorname{task}^{8}$. In that study, however, older subjects also performed more poorly on the task. In the present experiment, the simpler design of the MID task elicited equivalent performances from younger 
and older adults. Together, these findings suggest that ventral striatal activation during reward anticipation may not be as compromised by age as are the neural substrates recruited in the course of reversing reward associations (for example, ventrolateral prefrontal cortex ${ }^{17}$ ). Future research will have to specifically disentangle reward anticipation from reward reversal to fully test this possibility.

Although older adults did not differ significantly from younger adults during gain anticipation, they did differ during loss anticipation. Specifically, affective data indicated that older adults experienced less negative arousal (Supplementary Results), and neural data indicated that they showed less activation of the insula and caudate when exposed to loss cues. It is unlikely that the reduced neural activation during loss anticipation was a result of a general lack of response in these brain regions in older adults, as the same regions showed significant activation during gain anticipation. An asymmetry between positive and negative emotional experience has been documented in older adults in a number of behavioral studies using a variety of tasks ${ }^{18}$. Interpreted through the lens of socioemotional selectivity theory, age-related sparing of positive emotional experience may be related to efforts to optimize emotional experience as one approaches the end of life ${ }^{4}$. One aspect of this optimization may involve reducing negative arousal during anticipation of negative events. Notably, older adults did not show reduced neural responsiveness to loss outcomes themselves, as both older and younger adults had similar responses to loss outcomes (Supplementary Results). Although the present findings cannot establish whether reduced neural and affective responsiveness to loss anticipation results from effortful processing on the part of older adults, these findings are consistent with other reports indicating that older adults experience reduced negative emotion 19,20 .

Recent evidence distinguishes brain mechanisms involved in learning about positive and negative incentives ${ }^{21}$, and it is possible that older adults' reduced neural and affective responses during loss anticipation resulted from slower learning of the significance of loss cues, even though all participants received training on the task before scanning. However, a second experiment indicated that there were no differences between age groups in overall performance for learning of either gain or loss contingencies (Supplementary Results' Supplementary Table 3 and Supplementary Figs. 13 and 14 online). Reduced responsiveness to potential loss in the absence of cumulative learning deficits is consistent with a previous study comparing the performance of younger and older adults in a gambling task. That study 22 found that older adults performed as well as younger adults on a gambling task, despite showing skin conductance responses before choosing options associated with higher gains rather than losses 22 . Even after learning has taken place (or in the absence of learning), incentives still may vary in their impact. Regardless of the source, reduced responsiveness to anticipated loss may still have significant consequences for decision-making in older adults. Future research will have to explore this possibility.

Although an asymmetry in loss anticipation may enhance well-being in older adults, it may also engender biases in certain decision-making scenarios. For instance, risk assessment might be altered. Findings from this line of basic research may have implications for scientists' understanding of how processes underlying decision-making change with age, and might eventually facilitate the identification of markers for suboptimal decision-making in older adults $^{23}$.

\section{METHODS}

\section{Participants}

Twelve younger adults (age 19-27, six female) and 12 older adults (age 65-81, six female) participated in a MID task while undergoing fMRI. All participants gave written informed 
consent, and the experiment was approved by the Institutional Review Board of the Stanford University Medical School.

Care was taken to assess potential confounding baseline differences in both self-reports and neural activation between age groups. The two groups did not differ in years of education $(P$ $>0.05)$, in trait measures of affect $(P>0.05)$, in personality variables $(P>0.05)$ or in blood oxygen level-dependent (BOLD) signal amplitude $(P>0.05)$ as assessed by a visual localizer task (Supplementary Methods, Supplementary Table 4 and Supplementary Fig. 15 online).

\section{MID task}

A canonical version of the MID task ${ }^{24}$ was modified in two ways. First, the display duration of each frame of the task was lengthened to accommodate differences in vision and reading time among younger and older participants. Second, the traditionally used abstract symbolic cues (that is, closed circles and open squares) were replaced with literal symbolic cues (Win $\$ 0.00$, Win $\$ 0.50$, Win $\$ 5.00$, Lose $\$ 0.00$, Lose $\$ 0.50$, Lose $\$ 5.00$ ) that explicitly stated whether the trial was a potential gain or loss trial as well as the amount of money at stake. Across both runs, the entire task included 180 10-s trials. During each MID trial, participants viewed one of six different cues displaying the amount of money that could be gained or lost on that trial (anticipation phase). If the participant responded quickly enough to a subsequent target, he or she either gained or avoided losing money (outcome phase) (Supplementary Fig. 16 online). The six trial types were each presented 30 times ( 15 times per run) in an individually randomized order for each participant. The hit and miss rate for individual participants was manipulated by altering the average duration of the target with an adaptive timing algorithm that was originally set to the individual's mean reaction time in prescan practice, and then followed his or her performance across the scanned blocks, such that the individual would successfully hit the target on approximately $66 \%$ of the trials for each cue type. Individual functional volume acquisitions were time-locked to cue onsets using a drift adjustment algorithm, and thus coincided with each frame of the trials. After the MID task scan, participants rated their affective reactions to each of the cues on seven-point Likert scales (that is, valence from 'very negative' to 'neutral' to 'very positive' and arousal from 'not at all aroused' to 'highly aroused').

Hits were calculated as the percentage of correct responses per condition (that is, the button press occurred during target presentation). Ratings of cue-elicited valence and arousal were mean-deviated within individual across cues and plotted in a euclidean two-dimensional space. These dimensions were then rotated by $45^{\circ}$ to derive measures of positive arousal (PA; $\mathrm{PA}=\operatorname{arousal} / \sqrt{2}+$ valence $/ \sqrt{2}]$ and negative arousal (NA;

$\mathrm{NA}=\operatorname{arousal} / \sqrt{2}-$ valence $/ \sqrt{2}]$ (ref. 25). Actual hit rate, cue-elicited PA for gain cues and cue-elicited NA for loss cues were analyzed with mixed-model analyses of variance (ANOVAs) with incentive valence (gain, loss) and magnitude $(\$ 0.00, \$ 0.50, \$ 5.00)$ as withinsubject factors, and age (younger, older) as the between-subject factor. In the event of a significant interaction, PA and NA ratings were compared across all magnitude conditions for each group with within-subject ANOVAs (corrected for four comparisons, $P<0.013$ ) and direct comparisons were made between groups for each cue with between-subject $t$-tests (corrected for six comparisons, $P<0.008$ ). Further analyses isolated differences within groups in both valence and arousal using $t$-tests (corrected for eight comparisons, $P<0.006$ ).

\section{fMRI acquisition and analysis}

Imaging of the MID task was done using a 1.5-T General Electric MRI scanner with a standard quadrature head coil. Twenty-four 4-mm-thick slices (in-plane resolution, $3.75 \times 3.75 \mathrm{~mm}$; no gap) extended axially from the midpons to the top of the skull; this volume provided adequate spatial resolution of subcortical regions of interest (for example, midbrain, ventral striatum) 
while omitting only the base of the cerebellum or crown of the skull in some participants. Functional scans of the entire brain were acquired every $2 \mathrm{~s}$ (repetition time, $2 \mathrm{~s}$ ) with a T2*sensitive in-out spiral pulse sequence (echo time, $40 \mathrm{~ms}$; flip, $90^{\circ}$ ) specifically designed to minimize signal dropout at the base of the brain ${ }^{26}$. High-resolution structural scans were subsequently acquired using a T1-weighted spoiled gradient recalled acquisition in steady state sequence (repetition time, $100 \mathrm{~ms}$; echo time, $7 \mathrm{~ms}$; flip, 90 ${ }^{\circ}$ ), which facilitated subsequent localization and coregistration of functional data.

Analyses focused on changes in brain activation during anticipation (that is, after participants saw cues but before they responded to targets) and outcome (that is, after participants received feedback about their success and monetary gains/losses) for both gain and loss trials. All analyses were conducted using Analysis of Functional Neural Images software 27 . For preprocessing, voxel time series were concatenated across runs, sinc interpolated to correct for non-simultaneous slice acquisition within each volume and corrected for three-dimensional motion. Visual inspection of motion correction estimates confirmed that no subject's head moved more than $2 \mathrm{~mm}$ in any dimension from one volume acquisition to the next. Data were then bandpass filtered to admit frequencies between 10 and $90 \mathrm{~s}$, and the percentage signal change was calculated for each voxel with respect to the mean activation over the entire experiment.

Preprocessed time series data for each individual were analyzed with multiple regression 28 . The regression model consisted of a set of four orthogonal regressors of interest: gain $(\$ 0.50$, $\$ 5.00)$ versus nongain $(\$ 0.00)$ anticipation, loss $(\$ 0.50, \$ 5.00)$ versus nonloss $(\$ 0.00)$ anticipation, gain (hit: $\$ 0.50, \$ 5.00$ ) versus nongain (miss: $\$ 0.50, \$ 5.00$ ) outcome, and nonloss (hit: $\$ 0.50, \$ 5.00$ ) versus loss (miss: \$0.50, \$5.00) outcome. Additional covariates included two orthogonal regressors highlighting the periods of interest (anticipation and outcome), six regressors describing residual motion and six regressors modeling baseline, linear and quadratic trends for each experimental session. Regressors of interest were convolved with a gamma-variate function that modeled a prototypical hemodynamic response ${ }^{29}$ before inclusion in the regression model. Maps of $t$-statistics representing each of the regressors of interest were transformed into z-scores, slightly spatially smoothed to account for anatomical variability (kernel full-width half-maximum $=4 \mathrm{~mm}$ ), resampled at $2 \mathrm{~mm}^{3}$ and spatially normalized by warping to Talairach space. Statistical maps were then generated for the younger and older age groups using one-sample $t$-tests. Thresholds for statistical significance within the predicted volumes of interest (that is, striatum, anterior insula and mesial prefrontal cortex) were determined by a local small-volume correction (six 6-mm-diameter spheres or approximately ten $4-\mathrm{mm}^{3}$ voxels corrected at $P<0.05$, yielding a threshold $z$ of $2.81, P<$ 0.005 , uncorrected) and required a minimum cluster of eight face-to-face, contiguous $2-\mathrm{mm}^{3}$ resampled voxels. Thresholds for statistical significance outside of the predicted volumes of interest were set using a global family-wise error rate that corrected for gray matter volume in subcortical and mesial prefrontal cortical regions (approximately $5004-\mathrm{mm}^{3}$ voxels corrected at $P<0.05$, yielding a threshold $z$ of $3.89, P<0.0001$, uncorrected ${ }^{13}$ ) and required a minimum cluster of eight face-to-face, contiguous $2-\mathrm{mm}^{3}$ resampled voxels.

Group analyses consisted of two types: localization and decomposition. For the localization analyses, direct $t$-tests compared contrast coefficient maps within each group. The goal of the localization analysis was to verify that a priori regions of interest were activated in both age groups, as well as to identify new regions that might be correlated with regressors of interest for one group but not the other. For the decomposition analyses, VOIs were specified by imposing 6-mm-diameter spheres at foci defined a priori in regions of interest in the ventral striatum, medial caudate, anterior insula and mesial prefrontal cortex 24,30 . Care was taken to ensure that data from VOIs included only gray matter for each individual (see Supplementary Methods and Supplementary Table 5 online). Activation time courses were extracted and 
averaged from these VOIs by trial type. Peak anticipatory signal change (at a 6-s lag) was then compared using mixed-model ANOVAs with incentive valence (positive, negative), magnitude (\$0.00, $\$ 0.50, \$ 5.00)$ and subsequent outcome (hit, miss) as within-subject factors, and age group (younger, older) as the between-subject factor for each VOI. Outcome was included in the model to verify that signals extracted during the anticipatory period were not related to outcome activation. In the event of a significant interaction, values were compared across incentive and nonincentive conditions for each group using within-subject ANOVAs (corrected for four comparisons, $P<0.013$ ). Peak outcome signal change (at a 6-s lag) was also compared using mixed-model ANOVAs with incentive valence (positive, negative), magnitude (\$0.00, $\$ 0.50, \$ 5.00)$ and outcome (hit, miss) as within-subject factors, and age group (younger, older) as the between-subject factor for each VOI. In the event of a significant interaction, values were compared across hits and misses for incentive conditions (gain $\$ 0.50$, $\$ 5.00$ versus fail to gain $\$ 0.50, \$ 5.00$; avoid loss $\$ 0.50, \$ 5.00$ versus lose $\$ 0.50, \$ 5.00$ ) for each group with within-subject $t$-tests (corrected for four comparisons, $P<0.013$ ). No direct tests between groups for each of the individual six trial types were performed to avoid confounding differences in hemodynamic modulation between age groups as suggested by a recent review of BOLD imaging and aging ${ }^{31}$. Therefore, post hoc VOI analyses for both anticipation and outcome focused on linear effects within groups.

Correlational analyses assessed the relationship between self-reported anticipatory affect and anticipatory activation in the ventral striatum, medial caudate and anterior insula. A measure of cue-elicited affect change (PA, NA, valence and arousal) was computed by averaging selfreports for incentive cues $(\$ 0.50, \$ 5.00)$ and subtracting self-reports for nonincentive cues (\$0.00). Similarly, a measure of anticipatory activation change (ventral striatum, medial caudate and anterior insula) was computed by averaging the activation for incentive cues $(\$ 0.50, \$ 5.00)$ and subtracting activation for nonincentive cues $(\$ 0.00)$. The correlations between these change scores as reported are one-tailed as a result of our a priori directional hypotheses that activation during gain would correlate with PA and activation during loss would correlate with NA.

For methodological details of the follow-up behavioral learning study, see the Supplementary Methods and Supplementary Figure 17 online.

\section{Supplementary Material}

Refer to Web version on PubMed Central for supplementary material.

\section{Acknowledgements}

We would like to thank G.E. Wimmer and N.G. Hollon for assistance with data collection. This research was supported by US National Institute on Aging Research Grant AG008816, Center on the Demography and Economics of Health and Aging grant AG017253 and Center on Advancing Decision Making in Aging grant AG024957.

\section{References}

1. Park, DC.; Schwarz, N., editors. Cognitive Aging: A Primer. Psychology Press/Taylor \& Francis; Philadelphia: 2000.

2. Cabeza, R.; Nyberg, L.; Park, D. Cognitive Neuroscience of Aging: Linking Cognitive and Cerebral Aging. Oxford University Press; New York: 2005.

3. Carstensen, LL.; Mikels, JA.; Mather, M. Aging and the intersection of cognition, motivation and emotion. In: Birren, JE.; Schaie, KW., editors. Handbook of the Psychology of Aging. Academic Press; San Diego: 2005. p. 343-362.

4. Carstensen LL. The influence of a sense of time on human development. Science 2006;312:19131915. [PubMed: 16809530] 
5. Mather M, Carstensen LL. Aging and motivated cognition: the positivity effect in attention and memory. Trends Cogn Sci 2005;9:496-502. [PubMed: 16154382]

6. Knight, M.; Mather, M. The affective neuroscience of aging and its implications for cognition. In: Canli, T., editor. The Biological Bases of Personality and Individual Differences. Guilford Press; New York: 2006. p. 159-183.

7. Mather M, et al. Amygdala responses to emotionally valenced stimuli in older and younger adults. Psychol Sci 2004;15:259-263. [PubMed: 15043644]

8. Marschner A, et al. Reward-based decision-making and aging. Brain Res Bull 2005;67:382-390. [PubMed: 16216684]

9. Backman L, et al. Age-related cognitive deficits mediated by changes in the striatal dopamine system. Am J Psychiatry 2000;157:635-637. [PubMed: 10739428]

10. Kaasinen V, et al. Age-related dopamine D2/D3 receptor loss in extrastriatal regions of the human brain. Neurobiol Aging 2000;21:683-688. [PubMed: 11016537]

11. Raz, N. The aging brain observed in vivo: Differential changes and their modifiers. In: Cabeza, R.; Nyberg, L.; Park, D., editors. Cognitive Neuroscience of Aging: Linking Cognitive and Cerebral Aging. Oxford University Press; New York: 2005. p. 19-57.

12. Volkow ND, et al. Association between decline in brain dopamine with age and cognitive and motor impairment in healthy individuals. Am J Psychiatry 1998;155:344-349. [PubMed: 9501743]

13. Knutson B, Westdorp A, Kaiser E, Hommer D. fMRI visualization of brain activity during a monetary incentive delay task. Neuroimage 2000;12:20-27. [PubMed: 10875899]

14. Knutson B, Adams CM, Fong GW, Hommer D. Anticipation of increasing monetary reward selectively recruits nucleus accumbens. J Neurosci 2001;21:RC159. [PubMed: 11459880]

15. Bjork JM, et al. Incentive-elicited brain activation in adolescents: similarities and differences from young adults. J Neurosci 2004;24:1793-1802. [PubMed: 14985419]

16. Carstensen LL, Pasupathi M, Mayr U, Nesselroade JR. Emotional experience in everyday life across the adult life span. J Pers Soc Psychol 2000;79:644-655. [PubMed: 11045744]

17. Garavan H, Hester R, Murphy K, Fassbender C, Kelly C. Individual differences in the functional neuroanatomy of inhibitory control. Brain Res 2006;1105:130-142. [PubMed: 16650836]

18. Carstensen LL, Mikels JA. At the intersection of emotion and cognition: aging and the positivity effect. Curr Dir Psychol Sci 2005;14:117-121.

19. Kisley MA, Wood S, Burrows CL. Looking at the sunny side of life: age-related change in an eventrelated potential measure of the negativity bias. Psychol Sci. in the press

20. Wood S, Kisely MA. The negativity bias is eliminated in older adults: age related reduction in event related brain potentials associated with evaluative categorization. Psychol Aging 2006;21:815-820. [PubMed: 17201501]

21. Kim H, Shimojo S, O’Doherty JP. Is avoiding an aversive outcome rewarding? Neural substrates of avoidance learning in the human brain. PLoS Biol 2006;4:1453-1461.

22. Denburg NL, Recknor EC, Bechara A, Tranel D. Psychophysiological anticipation of positive outcomes promotes advantageous decision-making in normal older persons. Int J Psychophysiol 2006;61:19-25. [PubMed: 16426691]

23. Denburg NL, Tranel D, Bechara A. The ability to decide advantageously declines prematurely in some normal older persons. Neuropsychologia 2005;43:1099-1106. [PubMed: 15769495]

24. Knutson B, Fong GW, Bennett SM, Adams CS, Hommer D. A region of mesial prefrontal cortex tracks monetarily rewarding outcomes: characterization with rapid event-related fMRI. Neuroimage 2003;18:263-272. [PubMed: 12595181]

25. Knutson B, Taylor J, Kaufman M, Peterson R, Glover GH. Distributed neural representation of expected value. J Neurosci 2005;25:4806-4812. [PubMed: 15888656]

26. Glover GH, Law CS. Spiral-in/out BOLD fMRI for increased SNR and reduced susceptibility artifacts. Magn Reson Med 2001;46:515-522. [PubMed: 11550244]

27. Cox RW. AFNI: software for analysis and visualization of functional magnetic resonance neuroimages. Comput Biomed Res 1996;29:162-173. [PubMed: 8812068]

28. Neter, J.; Kutner, MH.; Nachtsheim, CJ.; Wasserman, W. Applied Linear Statistical Models. Irwin; Chicago: 1996. 
29. Cohen MS. Parametric analysis of fMRI data using linear systems methods. Neuroimage 1997;6:93103. [PubMed: 9299383]

30. Kuhnen CM, Knutson B. The neural basis of financial risk-taking. Neuron 2005;47:763-770. [PubMed: 16129404]

31. Gazzaley, A.; D’Esposito, M. BOLD functional MRI and cognitive aging. In: Cabeza, R.; Nyberg, L.; Park, D., editors. Cognitive Neuroscience of Aging: Linking Cognitive and Cerebral Aging. Oxford University Press; New York: 2005. p. 107-131. 


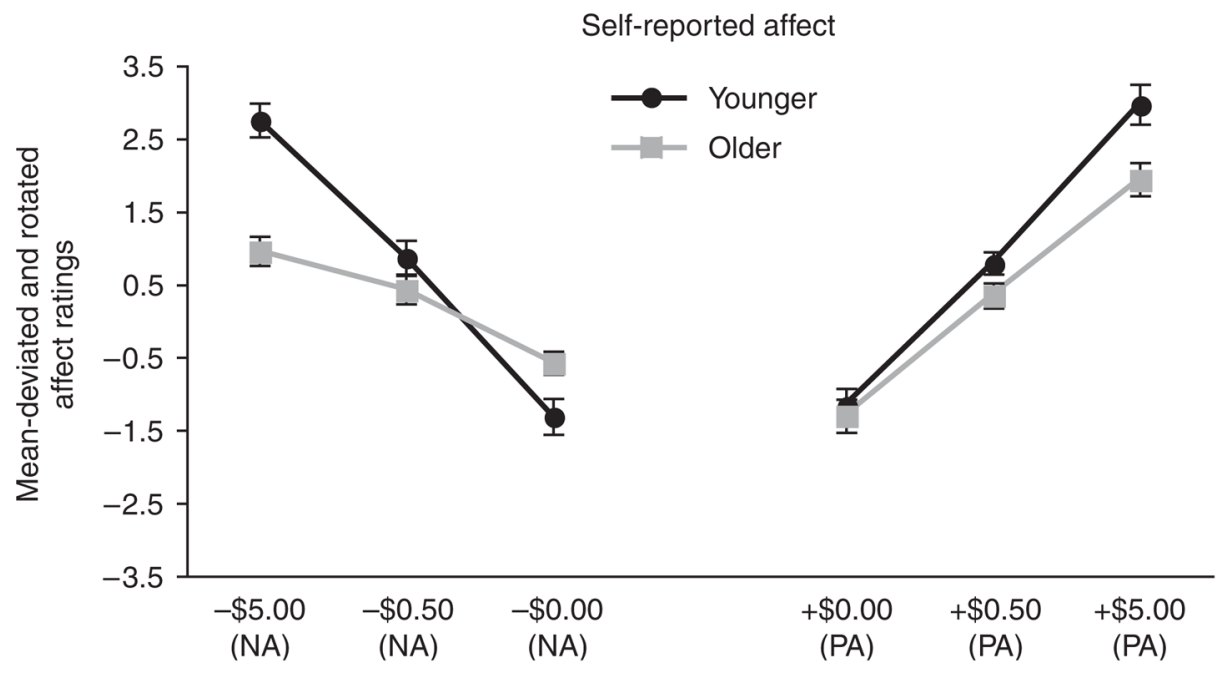

Figure 1.

Age by valence by magnitude interaction in post-task cue ratings. Younger adults self-reported monotonically increasing NA for loss cues and PA for gain cues in the anticipatory period. Older adults reported monotonically increasing PA for gain cues, but less significant increases in NA for loss cues. Error bars represent s.e.m. 


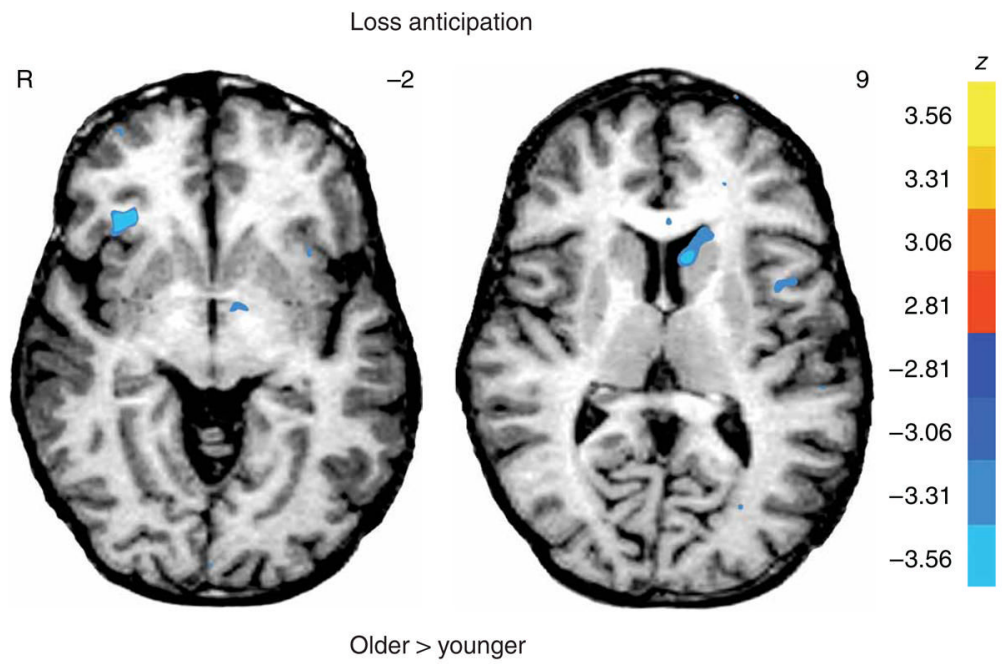

Figure 2.

Between-group $t$-tests of loss versus nonloss anticipation contrast maps (older adults $>$ younger adults; $\mathrm{SVC}, z>2.81 ; P<0.005$ uncorrected). Negative $z$-scores showed less activation for older adults in both the anterior insula and medial caudate. $S$ value for each axial image is listed in the upper right ( $S=-2$ through anterior insula; $S=9$ through striatum). 


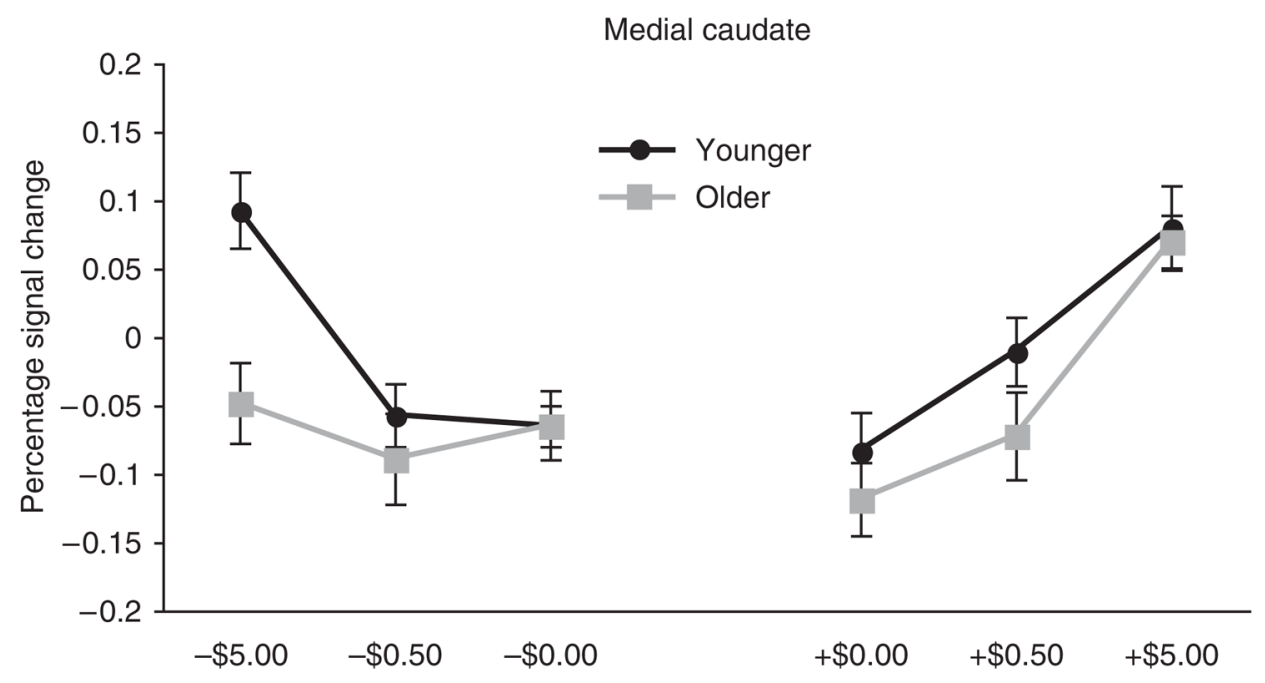

Figure 3.

BOLD activation extracted from the medial caudate at anticipation. An age by valence by magnitude interaction shows that younger adults had increasing activation for both gain and loss cues in the anticipatory period, but that older adults had increasing activation for gain, but not loss cues. Error bars represent s.e.m. See Supplementary Figure 8 for full activation time courses. 


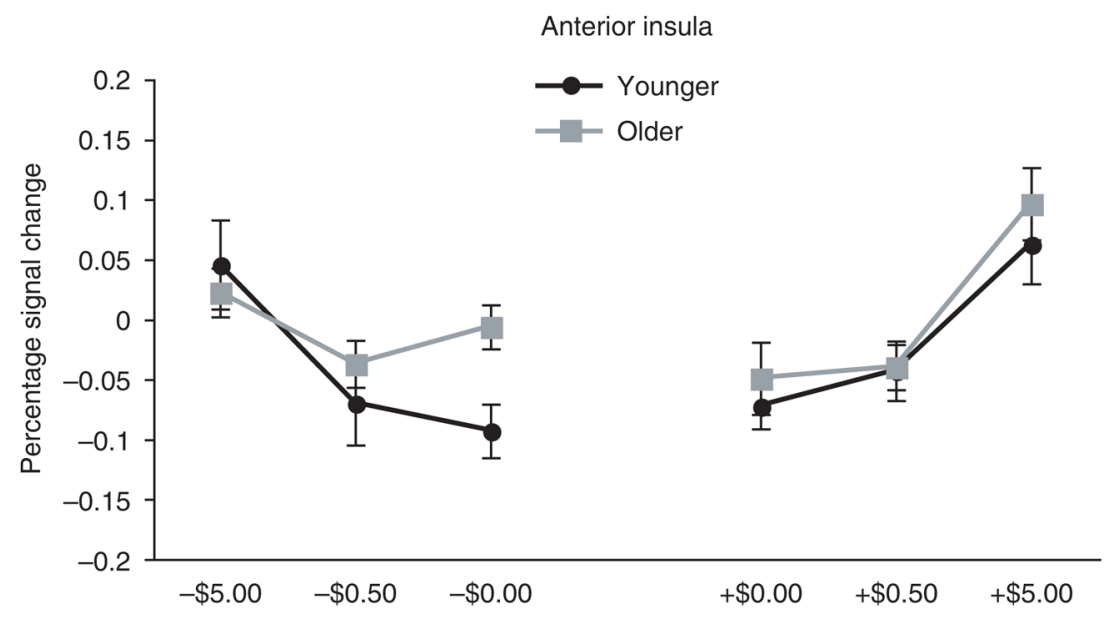

Figure 4.

BOLD activation extracted from the anterior insula at anticipation. An age by valence by magnitude interaction shows that younger adults had increasing activation for both gain and loss cues in the anticipatory period, but that older adults had increasing activation for gain but not loss cues. Error bars represent s.e.m. See Supplementary Figure 9 for full activation time courses. 